\title{
Rotation characteristic of a 3-DOF parallel mechanism with limbs of embedding structures
}

\author{
Gaowei Yang ${ }^{1, a}$, Jianjun Zhang ${ }^{1, b}$, Weimin $\mathrm{Li}^{1, \mathrm{c}}$, Kaicheng Qi ${ }^{1, \mathrm{~d}}$ \\ ${ }^{1}$ School of Mechanical Engineering, Hebei University of Technology, Tianjin 300130, China \\ aygw19841127@163.com, bzhjjun96@163.com, cvmin@163.com, dqkc@hebut.edu.cn
}

\begin{abstract}
Keywords: Parallel Mechanism, Continuous Rotation, Principal Screw.
Abstract. Three degrees of freedom (3-DOF) parallel mechanism (PM) with limbs of embedding structures is a PM that each limb exists common axis. This is an asymmetrical PM with a full cylinder workspace and it has two translational DOFs and one rotational DOF. In order to research the kinematics characteristic and rationally plan the motions of this PM, the rotation characteristic is studied based on screw theory. Firstly, principal screws of this PM are identified by quadric degenerating theory and the category of three-system is determined. The instantaneous kinematics is analyzed using the linear combinations of principal screws when this PM is under general configurations. Secondly, the change of the rotation of this PM under singular configuration is investigated by calculating the instantaneous DOFs. Finally, it is comprehensively analyzed that this PM maintains rotational DOF in any configurations, and can realize 360 degree continuous rotation under general configurations.
\end{abstract}

\section{Introduction}

A lower-mobility PM has fewer than six degrees of freedom. These PMs have lower cost than 6-DOF PMs, and are applied in the tasks where 6-DOF PMs are overqualified for the same tasks[1-2]. In lower mobility PMs, 3-DOF PMs have been studied extensively[3].

Therefore, the idea of new type of 3-DOF PMs has attracted several researchers. Many examples of 3-DOF PMs have been found in recent literatures: Torre and Rodriguez-leal presented a 3-CRC PM, and the instantaneous kinematic is analyzed by using the screw theory[4]. Ralpacelli designed a reconfigurable 3-DOF PM for multimodal tasks based on screw theory, Lie algebra and algebraic geometry[5]. Hou investigated a new full information modeling method to design a 3-DOF reconfigurable PM, and simulated its workspace. Zhao proposed a typed of synthesis approach for rotational parallel mechanisms with a virtual continuous axis[6]. Thereby, a great number of 3-DOF PMs have been proposed and studied. However, these PMs suffer from the shortcoming of smaller workspace. To overcome these defects, PM with limbs of embedding structures is proposed[7].

3-DOF PM with limbs of embedding structures is defined as PM with common axis between each limb. The interference between limbs is avoided and the workspace is increased by applying the embedding structure. In order to research the kinematic characteristics and plan the motions of moving platform, it is necessary to study the rotation characteristic.

Rotation characteristic includes two parts, one is instantaneous rotation when this PM is in general configurations, and the other is the change of rotation when this PM is in singular configuration. It is essential to analyze the instantaneous kinematics for understanding instantaneous rotation. Instantaneous kinematics is fundamental theoretical problem of parallel mechanism, and Zhao showed that the ideal tool to investigate the above problem is screw theory[8]. Huang presented a method for identification of the principal screws of three-order screw system based on the quadric degeneration theory. The method provides a detailed example for analyzing instantaneous kinematics[9]. This paper applies quadric degeneration theory to identify the principal screws of this PM, and the linear combination of principal screws is analyzed. Based on this analysis, it is determined that the moving platform can rotate 360 degree along a continuous axis when this PM is under general configurations. Then, the rotation of the moving platform is studied when this PM is in singular configuration. The result reveals that the rotation of moving platform is still maintained when 
this PM is in singular configuration. Finally, it is comprehensively analyzed that this PM maintains rotational DOF in any configuration, and can realize 360 degree continuous rotation around continuous axis under general configurations.

Based on the analysis of instantaneous motion and DOFs, besides the rotation characteristic, all the possible motions can be known when this PM is in general configurations. This result has the vital significance to the further research for this PM. This paper is arranged as follows: Section 2 presents a detailed description about this PM. Section 3 is the identification of principal screws and instantaneous kinematics analysis. Section 4 analyzes the rotation of the moving platform when this $\mathrm{PM}$ is in singular configuration. Section 5 is the conclusions.

\section{Architecture and Mobility}

The CAD model of the 3-DOF PM with limbs of embedding structures is shown in Fig.1 (a). The coordinate frame of the mechanism is shown in Fig.1 (b).

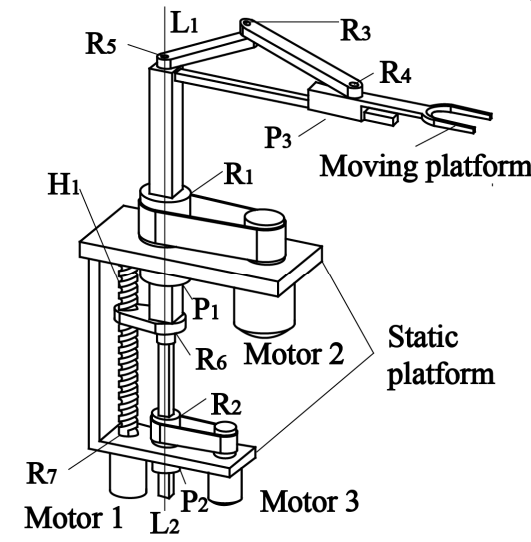

(a) CAD model

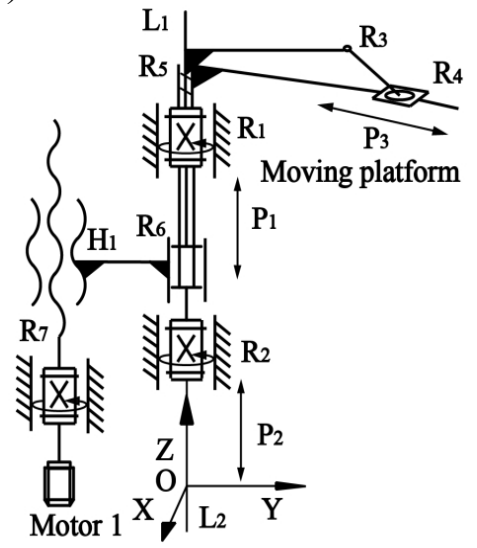

(b) The coordinate frame

Fig. 1. The 3-DOF PM with limbs of embedding structures

In Fig. 1 (b), $\mathrm{Z}$ axis is prismatic axis $\left(\mathrm{P}_{\mathrm{i}}\right.$ : prismatic joint and $\left.\mathrm{i}=1,2,3, \ldots, \mathrm{n}\right)$, and $\mathrm{O}$ is the origin located at the midpoint of $R_{2}$ joint $\left(R_{i}\right.$ : revolute joint and $\left.i=1,2,3, \ldots, n\right)$. In this $P M$, inputs $R_{1}, R_{2}$ and $\mathrm{R}_{7}$ are driven by motor 2 , motor 3 and motor 1 . This mechanism has three inputs, each of them works independently. The three DOFs are as follows: motor 1 drives moving platform moving along $\mathrm{Z}$ axis through $\mathrm{H}_{1}\left(\mathrm{H}_{\mathrm{i}}\right.$ : helical joint and $\left.\mathrm{i}=1,2,3, \ldots, \mathrm{n}\right)$ joint; motor 2 uses belts to operate moving platform rotating around $\mathrm{Z}$ axis; motor 3 drives crank rotating around $\mathrm{Z}$ axis, enabling moving platform radial movement centered on $\mathrm{Z}$ axis.

\section{Identification of Principle Screw and Instantaneous Kinematics Analysis}

According to screw theory, the key to determine instantaneous kinematics of a 3-DOF PM are both twist and the distribution of the twist axes in three-dimensional space. However, the main factor to determine the positions and orientations of all these screws in three-dimensional space is to get the principal screws of the screw system. So the principle screws are very important. The identification of principle screws consists of the following three steps:

(1) The screw system of each limb is established by imaginary mechanism[9].

(2) Based on the screw system of each limb, the first-order influence coefficient matrix is obtained.

(3) Substituting the first-order influence coefficient matrix into the formula of the distribution of the twists axes, and expanding the formula into the quadric surface formula. The principle screws are identified according to quadric degenerating theory and the characteristic.

After the principle screws are identified, the instantaneous kinematics is analyzed by the linear combination of principle screws, and the instantaneous rotation characteristic is determined when this $\mathrm{PM}$ is in general configurations.

Establish screw system of each limb. The screw systems of limbs are the basis for the analysis of PM by screw theory. However, 3-DOF PM with limbs of embedding structures adopts the embedding 
structure leading to non-unique limb division, and not each limb division can get the first-order influence coefficient matrix. Therefore, it is necessary to choose reasonable limb division. Based on analysis, the prototype is improved and the reasonable limb division consists of $\mathrm{P}_{1} \mathrm{R}_{1} \mathrm{P}_{3}$ limb (seen in Fig.2) and $P_{2} R_{2} R_{5} R_{3} R_{4}$ limb (seen in Fig.3). The inputs are $R_{1}, P_{2}$ and $R_{2}$.

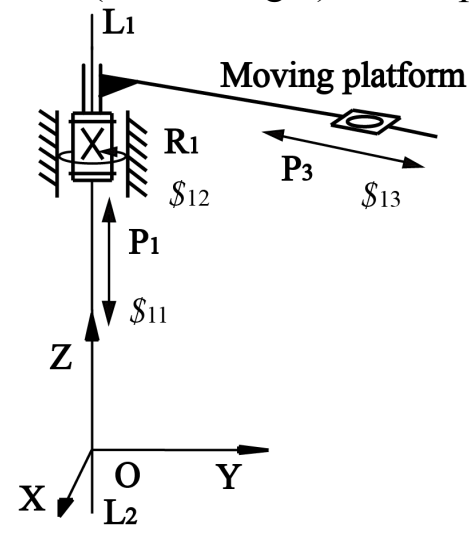

Fig.2. $\mathrm{P}_{1} \mathrm{R}_{1} \mathrm{R}_{3} \operatorname{limb}$

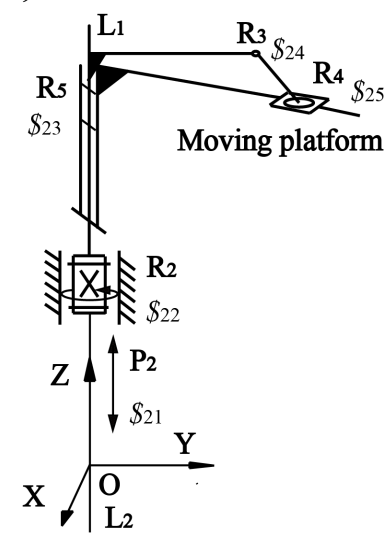

Fig.3. $\mathrm{P}_{2} \mathrm{R}_{2} \mathrm{R}_{5} \mathrm{R}_{3} \mathrm{R}_{4}$ limb

The twist system of $\mathrm{P}_{1} \mathrm{R}_{1} \mathrm{R}_{3}$ limb can be expressed as:

$$
\left\{\begin{array}{l}
\$_{11}=\left(S_{1} ; 0\right)=\left(\begin{array}{lllll}
0 & 0 & 1 ; 0 & 0 & 0
\end{array}\right) \\
\$_{12}=\left(0 ; S_{1}\right)=\left(\begin{array}{lllll}
0 & 0 & 0 ; 0 & 0 & 1
\end{array}\right) \\
\$_{13}=\left(0 ; S_{2}\right)=\left(\begin{array}{lllll}
0 & 0 & 0 ; x_{14} & y_{14} & z_{14}
\end{array}\right)
\end{array}\right.
$$

Where, coordinate $\left(x_{14}, y_{14}, z_{14}\right)$ is the coordinate of reference point $\mathrm{P}$ on the moving platform.

The twist system of $\mathrm{P}_{2} \mathrm{R}_{2} \mathrm{R}_{5} \mathrm{R}_{3} \mathrm{R}_{4}$ limb can be expressed as:

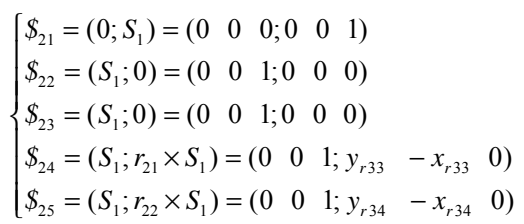

Where, $\left(x_{14} y_{14} z_{14}\right)=\left(x_{r 34} y_{r 34} z_{r 34}\right), S_{\mathrm{i}}$ and $r_{\mathrm{ij}}(\mathrm{i}, \mathrm{j}=1,2, \ldots, \mathrm{n})$ denote the unit vector of screw axis and the position vector pointing from the origin to an arbitrary point on the screw axis.

In order to establish the first-order influence coefficient matrix, the method of imaginary mechanism is applied to add imaginary joints. In $\mathrm{P}_{1} \mathrm{R}_{1} \mathrm{R}_{3} \operatorname{limb}$, the imaginary revolute joints(IRJs) are added as follows:

(a). An IRJ whose axes is perpendicular to $\mathrm{Y}$ axis and parallel to $\mathrm{Z}$ axis is denoted as $\mathrm{R}_{8}$. The distance between the axes of $\mathrm{R}_{8}$ and the origin is written as $B$.

(b). An IRJ whose axes coincides with $\mathrm{X}$ axis is denoted by $\mathrm{R}_{9}$.

(c). An IRJ whose axes is parallel to $\mathrm{Y}$ axis and perpendicular to $\mathrm{X}$ axis is denoted as $\mathrm{R}_{10}$. The distance between the axes of $\mathrm{R}_{10}$ and the origin is $B_{1}$.

The $P_{1} R_{1} R_{3}$ limb after adding imaginary joints is seen as Fig.4.

In $P_{2} R_{2} R_{5} R_{3} R_{4}$ limb, the IRJs are added as follows:

(a). An IRJ whose axes coincides with $\mathrm{X}$ axis is denoted by $\mathrm{R}_{11}$.

(b). An IRJ whose axes is parallel to $\mathrm{Y}$ axis and perpendicular to $\mathrm{X}$ axis is denoted as $\mathrm{R}_{12}$. The distance between the axes of $\mathrm{R}_{12}$ and the origin is written as $B_{1}$.

The $P_{2} R_{2} R_{5} R_{3} R_{4}$ limb after adding imaginary joints is seen as Fig.5.

The twist system of imaginary $\mathrm{P}_{1} \mathrm{R}_{1} \mathrm{R}_{3}$ limb is:

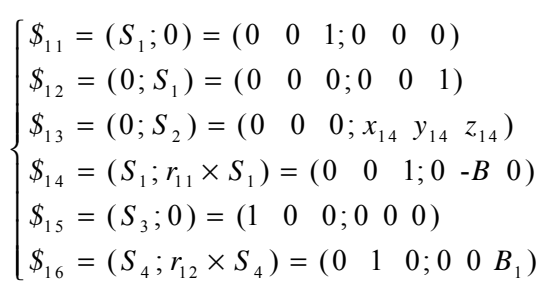




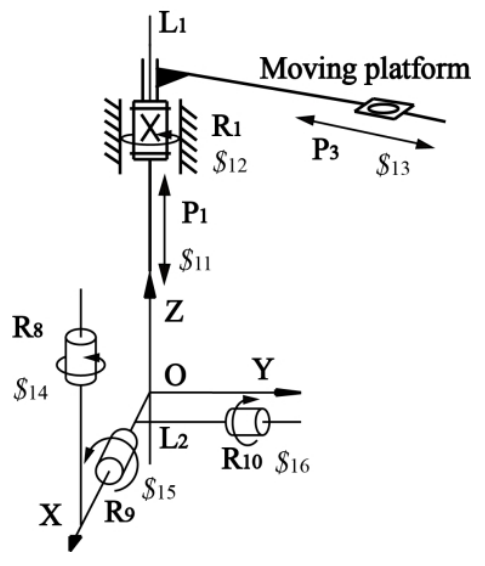

Fig.4. Imaginary $\mathrm{P}_{1} \mathrm{R}_{1} \mathrm{R}_{3} \operatorname{limb}$

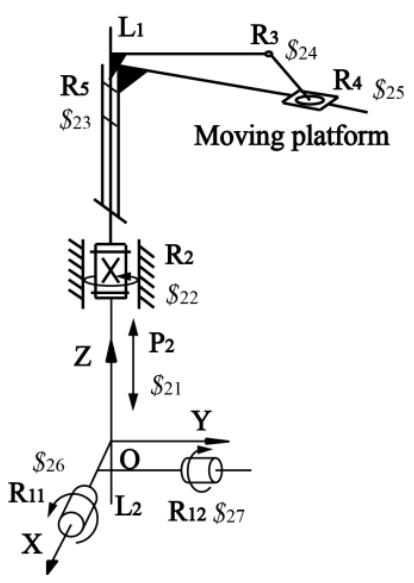

Fig.5. Imaginary $\mathrm{P}_{2} \mathrm{R}_{2} \mathrm{R}_{5} \mathrm{R}_{3} \mathrm{R}_{4}$ limb

In imaginary $P_{2} R_{2} R_{5} R_{3} R_{4}$ limb, $\$_{22}=\$_{23}$, so the twist system of this limb can be denoted as:

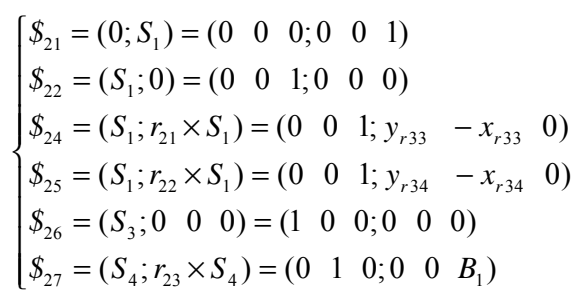

Brief introduction of calculation method of The first-order influence coefficient matrix. The screw systems of the two imaginary limbs can be expressed as:

$\left[G_{i}^{H}\right]=\left\{\$_{i 1}, \$_{i 2}, \$_{i 3}, \$_{i 4}, \$_{i 5}, \$_{i 6}\right\}, i=1,2$

Assume that the velocity of the moving platform is:

$$
V_{H}=\left\{\begin{array}{ll}
\omega & v_{P}
\end{array}\right\}^{T}
$$

Where $\omega$ is the angular velocity of the moving platform, $v_{P}$ is the linear velocity of the reference point $\mathrm{P}$. If the matrix $\left[G_{i}{ }^{H}\right]$ is no-singular, then:

$$
V_{H}=\left[G_{i}^{H}\right] \psi_{\text {III }} \quad i=1,2 j=1,2, \ldots, 6
$$

Where $\phi_{1}$ is the velocity of kinematic joints of each limb. Assume that the three inputs of this PM are \&, \&and $\&$, the velocity of each limb can be written as:

$$
\begin{aligned}
& \psi_{\gamma_{j}}=\left(\& \&, \&_{12}, \psi_{1}, 0,0,0\right)^{T}
\end{aligned}
$$

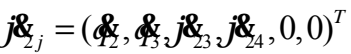

Inputs $\&$, $\&$ and $\&$ represent the angular velocity $\oint_{2}$ of $\mathrm{R}_{1}$, the angular velocity $\phi_{3}$ of $\mathrm{R}_{2}$ and the linear velocity $\Theta_{1}^{\oint}$ of $\mathrm{P}_{2}$. The inverse solution of Eq (7) is:

$$
\psi_{\mathrm{I}}=\left[G_{H}^{i}\right] V_{H}
$$

Where $\left[G_{H}^{i}\right]=\left[G_{i}^{H}\right]^{-1}$. Select the first row, the fifth row and the sixth row from $\left[G_{H}^{l}\right]$ and the first to third row from $\left[G_{H}^{2}\right]$ to form matrix $\left[G_{H}^{Q}\right]$. It follows that:

$$
\oint=\left[G_{H}^{Q}\right] V_{H}
$$

Where $\left[G_{Q}^{H}\right]=\left[G_{H}^{Q}\right]^{-1}$. Select the first three columns of $\left[G_{Q}^{H}\right]$ to form new matrix $\left[G_{q}^{H}\right]$.

$$
\left\{\begin{array}{l}
v_{p}=[G] \phi \\
\omega=\left[G^{\prime}\right] \phi
\end{array}\right.
$$

The $\mathrm{Eq}(12)$ denotes the corresponding relation of inputs and outputs of this PM, where the matrix $\left[G^{\prime}\right]$ is the first three rows of the matrix $\left[G_{q}^{H}\right]$, the matrix $[G]$ is the last three rows of the matrix $\left[G_{q}^{H}\right]$. 
Calculation of the first-order influence coefficient matrix. According to the above calculation method, the matrix of the first-order influence coefficient matrix $[G]$ and $\left[G^{\prime}\right]$ can be get:

$$
\begin{gathered}
{[G]=\left(\begin{array}{ccc}
M / N & -M / N & 0 \\
M_{1} / N & -M_{1} / N & 0 \\
0 & 0 & 1
\end{array}\right)} \\
{\left[G^{\prime}\right]=\left(\begin{array}{lll}
0 & 0 & 0 \\
0 & 0 & 0 \\
1 & 0 & 0
\end{array}\right)}
\end{gathered}
$$

Where, $M_{1}=\operatorname{arsin} \theta_{2} \sin \left(\theta_{2}-\theta_{3}\right), N=a \cos \left(\theta_{2}-\theta_{3}\right)-r\left(\theta_{2}\right.$ and $\theta_{3}$ are the rotation angles of $\mathrm{R}_{1}$ and $\left.\mathrm{R}_{2}\right)$. Parameters $a, b$ and $r$ are seen in Fig. 6.

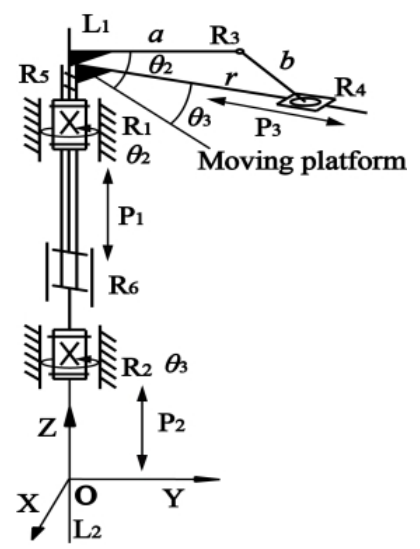

Fig.6. Illustration of 3-DOF parallel mechanism with limbs of embedding structures

Identification of principal screw of 3-DOF PM with limbs of embedding structures. According to screw theory, the twist of the moving platform can be presented as:

\section{$[A]=[r]\left[G^{\prime}\right]-[G]+h\left[G^{\prime}\right]$}

Where, $[r]$ is a skew-symmetric matrix whose elements indicate the coordinates of reference point ( $x y z$ ) on the twist axis of the moving platform in the coordinate system, $h$ is the pitch of output twist screw. If $\operatorname{det}[A]=0, \mathrm{Eq}(15)$ can be expressed as:

$$
c_{11} x^{2}+c_{22} y^{2}+c_{33} z^{2}+2 c_{12} x y+2 c_{23} x y+2 c_{13} x z+2 c_{14} x+2 c_{24} y+2 c_{34} z+c_{44}=0
$$

$\mathrm{Eq}(16)$ is a quadratic surface of $x, y$ and $z$, the coefficient $c_{\mathrm{ij}}(\mathrm{i}=1,2,3,4, \mathrm{j}=1,2,3,4)$ being functions of $h,[G]$ and $\left[G^{\prime}\right]$. According to quadric degenerating theory, the pitches of principle screws can be obtained from invariant $D$ of quadratic surface. $D$ is following as:

$$
D=\left|\begin{array}{ccc}
c_{11} & c_{12} & c_{13} \\
c_{12} & c_{22} & c_{23} \\
c_{13} & c_{23} & c_{33}
\end{array}\right|=0
$$

Expand the above invariant $D$, the following equation can be obtained as:

$$
a_{1} h^{3}+a_{2} h^{2}+a_{3} h+a_{4}=0
$$

The coefficient $a_{\mathrm{i}}(\mathrm{i}=1,2,3,4)$ is the functions of $c_{\mathrm{ij}}$. The roots of Eq (18) correspond to pitches of principle screws of this PM. Based on matrix $\left[G^{\prime}\right]$ and $[G]$, the matrix $A$ can be expressed as:

$$
[A]=\left[\begin{array}{ccc}
y-m \cos \theta_{2} & m \cos \theta_{2} & 0 \\
-x-m \sin \theta_{2} & m \sin \theta_{2} & 0 \\
h & 0 & -1
\end{array}\right]
$$

Where $m=\operatorname{arsin}\left(\theta_{2}-\theta_{3}\right) /\left[a \cos \left(\theta_{2}-\theta_{3}\right)-r\right]$. The determinant of matrix $A$ is:

$\operatorname{det}[A]=m x \cos \theta_{2}-m y \sin \theta_{2}$ 
The Eq (20) is a linear equation, and the coefficient $c_{\mathrm{ij}}=0$. So the determinant of matrix $D$ is identically zero, and the pitches of principle screws are any value when this PM is under general configurations, namely $h_{\alpha}=h_{\beta}=h_{\gamma}=\infty$ ( $h_{\alpha}, h_{\beta}$ and $h_{\gamma}$ denote the pitches of three principle screws). In this case, it is necessary to consider actual situation of this PM. This PM has two translational DOFs and one rotational DOF. The translational DOFs are moving platform moving along $\mathrm{Z}$ axis and radial movement around $\mathrm{Z}$ axis. The rotational DOF is moving platform rotating around $\mathrm{Z}$ axis. The radial movement of the moving platform can be decomposed into movement along $X$ axis and $Y$ axis. The movement along $\mathrm{Z}$ axis and rotation around $\mathrm{Z}$ axis can be regarded as a helical motion along $\mathrm{Z}$ axis. Therefore, the pitches of the principle screws are $h_{\alpha}=h_{\beta}=\infty, h_{\gamma}=h$, where $h$ is a finite value. The principal screws are:

$$
\begin{aligned}
& \$_{\alpha}=\left(\begin{array}{lllll}
0 & 0 & 0 ; 1 & 0 & 0
\end{array}\right) \\
& \$_{\beta}=\left(\begin{array}{lllll}
0 & 0 & 0 ; 0 & 1 & 0
\end{array}\right) \\
& \$_{\gamma}=\left(\begin{array}{lllll}
0 & 0 & 1 ;
\end{array} \mathbf{0} 0 \begin{array}{ll}
0 & h_{\gamma}
\end{array}\right)
\end{aligned}
$$

According to Eq (21)-(23), the twist screw system under general configurations of this PM is the fifth special three-system[9]. The linear combination of principal screws of this PM is as follows:

$$
\begin{aligned}
& \$_{\Sigma}=\$_{\gamma}+\lambda_{1} \$_{\alpha}+\lambda_{2} \$_{\beta}=\left\{\begin{array}{llll}
0 & 0 & 0 ; \lambda_{1} & \lambda_{2} h_{\gamma}
\end{array}\right\} \\
& \$_{\Sigma 1}=\lambda_{3} \$_{\alpha}+\lambda_{4} \$_{\beta}=\left\{\begin{array}{lll}
0 & 0 & 0
\end{array} \lambda_{3} \lambda_{4} 0\right\}
\end{aligned}
$$

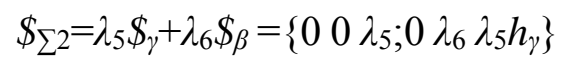

$$
\begin{aligned}
& \$_{\Sigma 3}=\lambda_{7} \$_{\alpha}+\lambda_{8} \$_{\gamma}=\left\{\begin{array}{lllll}
0 & 0 & \lambda_{8} ; \lambda_{7} & 0 & \lambda_{8} h_{\gamma}
\end{array}\right\}
\end{aligned}
$$

Where $\lambda_{i}(i=1,2 \ldots, 8)$ is any value.

Instantaneous kinematics analysis. Linear combination $S_{\Sigma}$ whose pitch is $h_{\gamma}$ denotes a helical motion along $Z$ axis. This PM has the same principal screw under general configurations.

Linear combination $S_{\Sigma 1}$ denotes translational motions in XY plane.

Linear combination $S_{\Sigma^{2}}$ and $S_{\Sigma_{3}}$ whose axes parallel to $\mathrm{Z}$ axis and their pitches are $h_{\gamma}$ denote the screw motion in $\mathrm{XZ}$ and $\mathrm{YZ}$ plane. The vector unit indicates the direction of the axes of $S_{\sum^{2}}$ and $S_{\sum_{3} 3}$ is the same as $S_{\Sigma}$.

The motions mentioned above can be realized when this PM is under general configurations. According to analysis of $S_{\Sigma}, S_{\Sigma^{2}}$ and $S_{\Sigma_{3}}$, this PM can realize 360 degree continuous rotation along Z axis.

\section{The rotational DOF of 3-DOF PM with limbs of embedding structures under singular configuration}

The principal screw analysis shows that the moving platform of this PM can realize 360 degree continuous rotation around $\mathrm{Z}$ axis. However, in order to research rotation characteristics, it still needs to consider the change of rotational DOF when this PM is under singular configuration.

There may exist two singular configurations in this PM, which are dead point and extremely displacement singularity respectively(seen in Fig.8). The dead point singularity can be avoided by adjusting component geometric parameters, but the extremely displacement singularity is difficult to avoid in practical work. Therefore, it is necessary to analyze extremely displacement singularity.

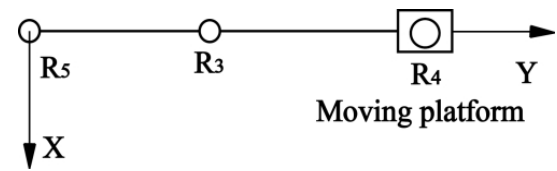

Fig.7. Extremely displacement singularity 
When this PM is in extremely displacement singularity, the moving platform can not move forward and only can move backward. Meanwhile, the kinematic joint $\mathrm{R}_{5}, \mathrm{R}_{3}$ and $\mathrm{R}_{4}$ are collinear, so the three joints are linear correlation. The screw systems of each limb are as follows:

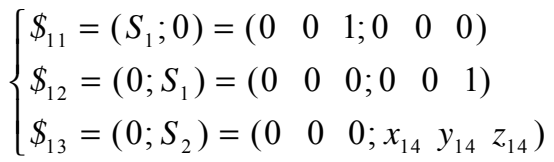

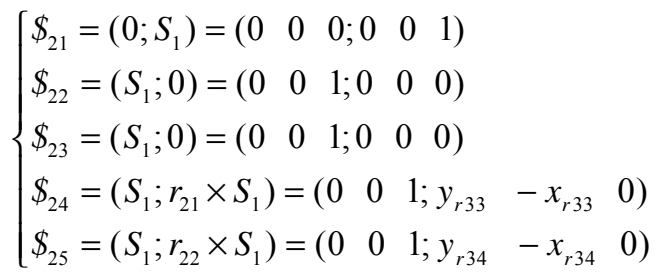

Where $\left(\begin{array}{lll}x_{14} & y_{14} & z_{14}\end{array}\right)=\left(\begin{array}{llll}x_{r 34} & y_{r 34} & z_{r 34}\end{array}\right)=\left(\begin{array}{lll}k x_{r 33} & k y_{r 33} & k z_{r 33}\end{array}\right), k$ is a real number. The $\operatorname{Eq}(29)$ can be simplified as:

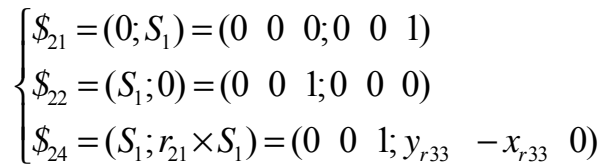

Based on Eq (28) and (30), the DOFs of this PM can be denoted as:

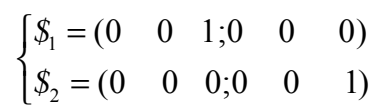

In general configurations, the DOFs of this PM can be denoted as:

$$
\left\{\begin{array}{l}
\$_{1}=\left(\begin{array}{lllll}
0 & 0 & 1 ; 0 & 0 & 0
\end{array}\right) \\
\$_{2}=\left(\begin{array}{lllll}
0 & 0 & 0 ; 0 & 0 & 1
\end{array}\right) \\
\$_{3}=\left(\begin{array}{lllll}
0 & 0 & 0 ; x_{14} & y_{14} & 0
\end{array}\right)
\end{array}\right.
$$

By contrasting Eq (31) and (32), when this PM is under extremely displacement singularity, the radial movement DOF is lost, and the rotational DOF is maintained. Comprehensive analysis of linear combination of principle screws shows that this PM can rotate in any configurations.

\section{Conclusions}

In order to research rotation characteristics of 3-DOF PM limbs of embedding structures, the rotation is analyzed when this PM is in general and singular configurations. It is determined that this PM can rotate in any configurations, and can realize 360 degree continuous rotation around $\mathrm{Z}$ axis under general configurations. At this point the following conclusions are obtained:

(1) The twist screw system under general configurations of this PM is always the fifth special three-system.

(2) When this PM is in extremely displacement singularity, the radial movement is lost, instead, the rotation and movement along $\mathrm{Z}$ axis are maintained.

\section{Acknowledgements}

This work is supported by the National Natural Science Foundation of China (Grant No.51175114) and Science and Technology Plan Project of Hebei Province of China(Grant No. 15211829).

\section{References}

[1]. PK. Jamwal: Forward kinematics modeling of a parallel ankle rehabilitation robot using modified 
fuzzy inference. Mechanism and Machine Theory. Vol.45(11), p.1537-1554 (2010).

[2]. Mohsen. Asgari, Mahdi A, Ardestani: Trajectory tracking control of a class of lower mobility parallel manipulators using ANFIS. ( IEEE Computer Society, Iranian 2013).

[3]. MC. Palpacelli: Analysis and design of a reconfigurable 3-DOF parallel manipulator for multimodal tasks (IEEE/ASME Transactions on Mechatronics, America 2014).

[4]. Zhen. Huang: submitted to International Journal of Robotics Research (2003).

[5]. Hussein. Torre, Ernesto. Rodriguez-Leal: submitted to International journal of advanced robotic system (2016).

[6]. Tieshi. Zhao: submitted to Mechanism and Machine Theory (2017).

[7]. Jianjun. Zhang, in: Parallel robotics, edtied by Jinsong.Wang, Xinjun, Liu. Nova Science Publisher, New York (2008), in press.

[8]. Yuefa. Fang: submitted to China Mechanical Engineering (2000).

[9]. Zhen. Huang: submitted to Mechanism and Machine Theory Vol. 8(36), p. 893-911(2001). 